\title{
The Zinc Story under High Pressure
}

\author{
Kenichi Takemura \\ Institute of Materials Structure Science (IMSS), High Energy Accelerator Research Organization (KEK), Tsukuba, Japan \\ Email: takemura@post.kek.jp
}

How to cite this paper: Takemura, K. (2019) The Zinc Story under High Pressure. Journal of Minerals and Materials Characterization and Engineering, 7, 354-372.

https://doi.org/10.4236/jmmce.2019.75024

Received: August 14, 2019

Accepted: September 23, 2019

Published: September 26, 2019

Copyright $\odot 2019$ by author(s) and Scientific Research Publishing Inc. This work is licensed under the Creative Commons Attribution International License (CC BY 4.0).

http://creativecommons.org/licenses/by/4.0/

\begin{abstract}
A historical review is presented on the experimental and theoretical studies on $\mathrm{Zn}$ under high pressure. Based on our high-pressure powder x-ray diffraction experiments that have been done for nearly a decade, we describe the structural change of $\mathrm{Zn}$ up to $126 \mathrm{GPa}$ at room temperature. Although several experimental and theoretical studies indicated an anomalous change of the $c / a$ axial ratio with pressure, we found no such an anomaly within our experimental uncertainty. Our high-pressure low-temperature experiments up to $18 \mathrm{GPa}$ at $40 \mathrm{~K}$ also gave no evidence of the $c / a$ anomaly. We suspect that the pressure-transmitting media played an important role in producing the anomaly. The structural anisotropy of $\mathrm{Zn}$ is drastically reduced at high pressures, which would be a general trend for hexagonal close-packed (hcp) metals.
\end{abstract}

\section{Keywords}

Zinc, High Pressure, Crystal Structure, Electronic Topological Transition

\section{Introduction}

Zinc is one of the elemental metals and used for many practical applications in industry [1]. It is an important constituent of various kinds of alloys such as brass and nickel silver. From a crystallographic point of view, zinc is unique among elemental metals. It takes hexagonal close-packed (hcp) structure, which is common for the crystal structure of metals. However, the ratio of the crystallographic axes $c$ to $a$ is quite large, being 1.856, far from the usual values of 1.57 1.65 [2]. Since the axial ratio $c / a$ of a hexagonal crystal with an ideal packing of rigid spheres is $1.633(\sqrt{8 / 3})$, it follows that the arrangement of atoms is not "close-packed" in zinc. Figure 1 schematically shows the atomic arrangement for the hcp structures with different $c / a$ axial ratios. Zinc belongs to the group shown on the right side. Due to the long $c$-axis, each zinc atom has six nearest neighbors in the $c$-plane and six next nearest neighbors in the adjacent planes. 


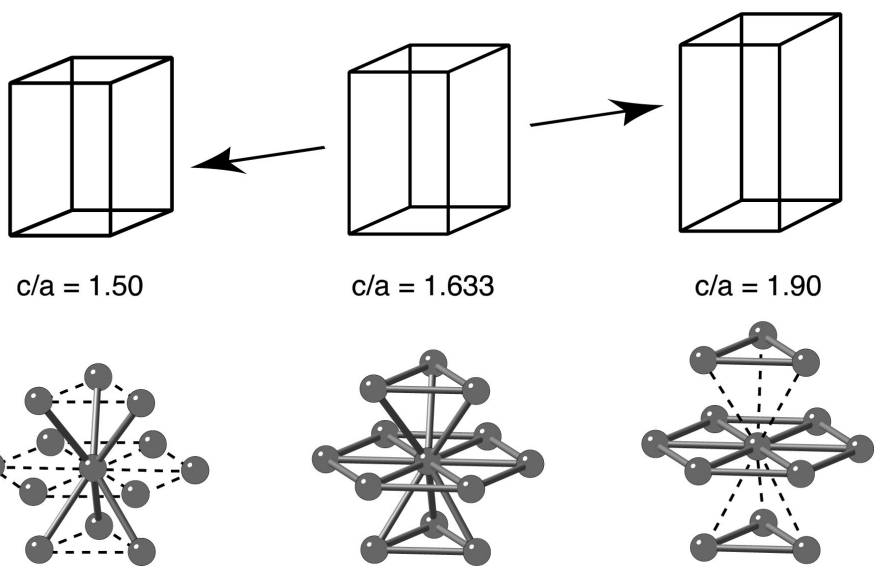

Figure 1. Hcp structures with three different $c / a$ axial ratios. The top figures show the hexagonal unit cells and the bottom figures show the atomic arrangement. The first nearest neighbors are connected by thick bonds while the second nearest neighbors are connected by broken lines.

Cadmium, another member of the group 12 elements, has also an hcp structure with a large $c / a$ axial ratio, 1.886. Mercury, the last member of the group 12 elements, is liquid under ambient conditions but takes a similar hcp structure in one of the solid phases formed under high pressure [3] [4]. All the group 12 elements can thus be viewed as anisotropic metals. Their physical properties like thermal expansion are highly anisotropic [5] [6].

A simple question then arises: What are the upper and lower limits for the $c / a$ axial ratio of the hcp structure? High-pressure is a powerful experimental tool to modify the crystal structure of substances. A number of high-pressure studies have been done on zinc specifically with a focus on its effect on the structural anisotropy. The purpose of the present article is to provide a historical review of the high-pressure studies on $\mathrm{Zn}$ and specifically to describe the detailed structural change under high pressure. Many theoretical calculations predict an electronic topological transition (ETT), which involves a change in the topology of the Fermi surface, occurring in $\mathrm{Zn}$ under high pressure. We discuss possible correlation between the change of crystal structure and electronic structure.

\section{Historical Review}

Table 1 summarizes high-pressure experimental studies on $\mathrm{Zn}$ to date. In 1940's Bridgman studied the compressibility of $\mathrm{Zn}$ by using a piston-cylinder apparatus from an interest in the behavior of elements under high pressure and obtained the pressure-volume relationship of $\mathrm{Zn}$ up to $10 \mathrm{GPa}$ [7]. Vaidya and Kennedy investigated more detailed pressure-volume relationship of $\mathrm{Zn}$ in the pressure range up to $4.5 \mathrm{GPa}$ [8]. Compression to extreme pressure and temperature conditions was also achieved by shock wave techniques in 1960's [9] [10]. The pressure-temperature phase diagram of $\mathrm{Zn}$ was studied by using a piston-cylinder apparatus [11] and most recently by a diamond-anvil cell coupled with laser-heating techniques [12]. In mid 1960's several groups were interested 
Table 1. High-pressure experimental studies on $\mathrm{Zn}^{\mathrm{a}} . P_{\max }$ is the highest pressure reached in each experiment.

\begin{tabular}{|c|c|c|c|}
\hline Measurement & Authors & $P_{\max }(\mathrm{GPa})$ & Ref. \\
\hline \multirow{2}{*}{$\begin{array}{l}\text { Volume compression by a } \\
\text { piston-cylinder apparatus }\end{array}$} & Bridgman (1941) & 10 & [7] \\
\hline & Vaidya \& Kennedy (1970) & 4.5 & [8] \\
\hline \multirow{2}{*}{ Shock compression } & McQueen \& Marsh (1960) & 140 & [9] \\
\hline & Al'tshuler et al. (1962) & 786 & [10] \\
\hline \multirow{2}{*}{ Melting and P-T phase diagram } & Akella et al. (1973) & 6 & {$[11]$} \\
\hline & Errandonea et al. (2018) & 140 & {$[12]$} \\
\hline \multirow{2}{*}{ Magnetoresistance } & Gaidukov \& Itskevich (1964) & 0.8 & {$[13]$} \\
\hline & Schirber (1965) & 0.6 & [14] \\
\hline de Haas-van Alphen oscillations & O’Sullivan \& Schirber (1966) & 0.4 & [15] \\
\hline \multirow{2}{*}{ Electrical resistance } & Lynch \& Drickamer (1965) & 50 & {$[16]$} \\
\hline & Garg et al. (2002) & 25 & [17] \\
\hline \multirow{10}{*}{$\begin{array}{c}\text { Crystal structure by } \\
\text { owder x-ray diffraction }\end{array}$} & Lynch \& Drickamer (1965) & 16 & [16] \\
\hline & McWhan (1965) & 10 & {$[18]$} \\
\hline & Schulte et al. (1991) & 30 & [19] \\
\hline & Takemura (1995) & 126 & [20] \\
\hline & Schulte \& Holzapfel (1996) & 74 & [21] \\
\hline & Takemura (1997) & 126 & [22] \\
\hline & Takemura (1999) & 21 & [23] \\
\hline & Takemura et al. $(2002,2002)$ & 18 & [24] [25] \\
\hline & Takemura et al. (2002) & 123 & {$[26]$} \\
\hline & Errandonea et al. (2018) & 140 & {$[12]$} \\
\hline \multirow{2}{*}{ Mössbauer spectroscopy } & Potzel et al. (1995) & 16 & [27] \\
\hline & Steiner et al. (1996) & 16 & [28] \\
\hline \multirow{2}{*}{ Inelastic neutron scattering } & Morgan et al. (1996) & 8.8 & [29] \\
\hline & Klotz et al. (1998) & 9.4 & [30] \\
\hline \multirow{2}{*}{ Raman scattering } & Olijnyk (1992) & 54 & {$[31]$} \\
\hline & Olijnyk et al. (2000) & 58 & {$[32]$} \\
\hline $\mathrm{x}$-ray absorption spectroscopy & Aquilanti et al. (2007) & 30 & [33] \\
\hline
\end{tabular}

${ }^{a}$ This list may not cover all the references specifically older ones. Please refer to each reference for finding old data sources.

in the effect of pressure on the energy band structure and the Fermi surface of Zn. They measured, for example, magneto resistance [13] [14] and de Haas-van Alphen oscillations [15] at high pressure and low temperature. These electrical measurements were limited to relatively low pressures up to about $1 \mathrm{GPa}$.

Lynch and Drickamer extended the pressure range of electrical measurement to $50 \mathrm{GPa}$ and reported an irregular change of the electrical resistance around 10 $\mathrm{GPa}$ [16]. They also studied the lattice parameters of $\mathrm{Zn}$ under pressure and found a hump in the change of the $c / a$ axial ratio in the same pressure range, where the electrical resistance showed the irregular behavior. They argued the origin of this anomaly based on the interaction of the Fermi surface with the Brillouin zone boundaries [34]. This is the first report, which indicated that something anomalous might be happening in $\mathrm{Zn}$ under high pressure. Later x-ray diffraction experiment by McWhan [18], on the other hand, gave no indi- 
cation of anomalous behavior in the change of the lattice parameters up to 10 GPa. After a blank of high-pressure research on $\mathrm{Zn}$ for about three decades, new measurements sparked again the interest on the anomaly in $\mathrm{Zn}$ under high pressure. A Mössbauer spectroscopy measurement by Potzel et al. [27] showed that the lattice dynamics of $\mathrm{Zn}$ drastically changed at about 6.6 GPa at low temperature [28]. They discussed the anomaly in terms of an ETT that is also known as the Lifshitz transition [35]. Soon after this report, based on powder x-ray diffraction experiments, Takemura reported an anomaly in the pressure dependence of the $c / a$ axial ratio of $\mathrm{Zn}$ at about $9 \mathrm{GPa}$ at room temperature [20]. $\mathrm{He}$ discussed the anomaly in correlation with the ETT suggested by the Mössbauer experiment. Since the axial ratio took a special value of $\sqrt{3}$ at this anomaly, he suggested another possibility that the elastic properties of hcp crystals may change in general at $c / a=\sqrt{3}$ [20] [22]. These two experimental studies, Mössbauer and x-ray diffraction, motivated a number of theoretical studies on the change of the electronic structure of $\mathrm{Zn}$ under high pressure. Some of them reproduced the anomaly in the pressure-dependence of the axial ratio, while others did not. We will discuss in detail about the comparison of experimental results with theoretical calculations in Section 4. Inelastic neutron scattering experiments were done to investigate the phonon dynamics of $\mathrm{Zn}$ [29] [30]. The experiment by Morgan et al. [29] indicated an anomaly occurring above $6.8 \mathrm{GPa}$, while another measurement by Klotz et al. [30] showed no such evidence and casted doubts on the existence of ETT in Zn.

In the meantime, Takemura reported the results of new powder $\mathrm{x}$-ray diffraction experiment on $\mathrm{Zn}$, which was performed with a helium pressure-transmitting medium (PTM). The new data gave no evidence of anomaly in the $c / a$ axial ratio [23]. He argued that the anomaly was most probably induced by the solidification and rapid hardening of the PTM used in his previous experiments that was a methanol-ethanol-water mixture. The new experiment used He as a PTM, which has the highest solidification pressure ( $11.5 \mathrm{GPa}$ at room temperature) among known PTM. Even after solidification, solid He offers quasi-hydrostatic stress conditions, providing negligible effect of nonhydrostatic stress [36]. The smooth change of the $c / a$ axial ratio seemed to rule out any anomaly at least in the crystal structure. However, the existence or nonexistence of ETT is another story. A number of theoretical calculations have been published. Experimental efforts continue to prove ETT in Zn by using other techniques [31] [32] [33]. A recent work [17] reported an irregular change of the electrical resistance of $\mathrm{Zn}$ around $10 \mathrm{GPa}$ similar to that observed by Lynch and Drickamer [16]. An x-ray diffraction study again suggested the existence of the $c l a$ anomaly [12].

\section{Crystal Structure}

In this section, we summarize the results of our structural study on $\mathrm{Zn}$ under high pressure that have been published elsewhere [20] [22]-[26]. We have carried out a series of powder $\mathrm{x}$-ray diffraction experiments on $\mathrm{Zn}$ at high pressures 
at room $(297 \mathrm{~K})$ and low $(40 \mathrm{~K})$ temperatures (see Appendix for the numerical data of our experiments). We used diamond-anvil cells for pressure generation. A fine powder of $\mathrm{Zn}$ was enclosed in a gasket together with various PTM in order to check the effect of nonhydrostaticity. We used a 4:1 mixture by volume of methanol and ethanol (ME) [37] or a 16:3:1 mixture of methanol, ethanol and water (MEW) [38], or helium [36] [39]. Pressure was determined with the ruby luminescence method. We used the pressure scale given by Mao et al. [40] for ME- and MEW-PTM, and that given by Zha et al. [41] for He-PTM. Powder $\mathrm{x}$-ray diffraction experiments have been performed on the beam lines $6 \mathrm{~B}, 14 \mathrm{C}$, and 18C of the Photon Factory, KEK. See [22]-[26] for more details of our experimental procedures.

Figure 2 shows the variation of the lattice parameters $a$ and $c$ with pressure at room temperature. The $c$-axis is much more compressible than the a-axis at low pressures, having linear compressibility of about eight times larger than that of the $a$-axis [5]. At higher pressures, the compressibility of the $c$-axis becomes comparable to that of the a-axis. As a consequence, the $c / a$ axial ratio rapidly decreases on the initial stage of compression and then decreases more slowly at higher pressures (Figure 3). There are systematic differences in the change of the axial ratio depending on the PTM used in the experiment. The discrepancy seems to come from the difference in the stress conditions of different PTM. In order to remove the uncertainty in pressure determination, we plot the change of the axial ratio as a function of relative volume $V / V_{0}$, where $V_{0}$ denotes the volume at atmospheric pressure (Figure 4). The discrepancy however still remains. Since the change of the axial ratio is smooth and the scatter is smaller for the

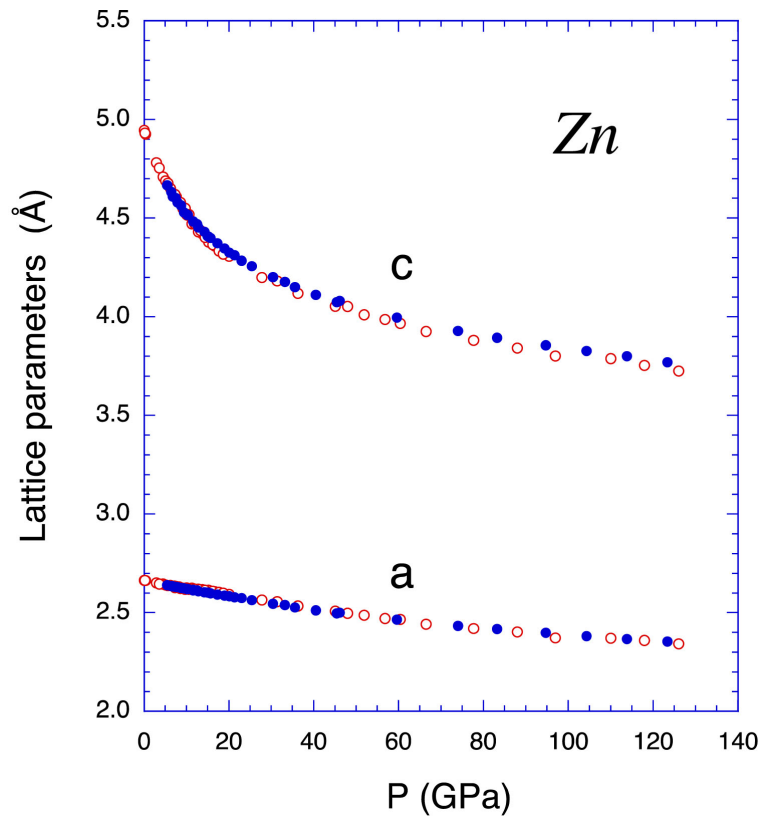

Figure 2. The change with pressure of the lattice parameters $a$ and $c$ of $\mathrm{Zn}$ at room temperature. Blue closed circles show the data taken with a He-PTM, while red open circles are taken with a ME- or MEW-PTM. 


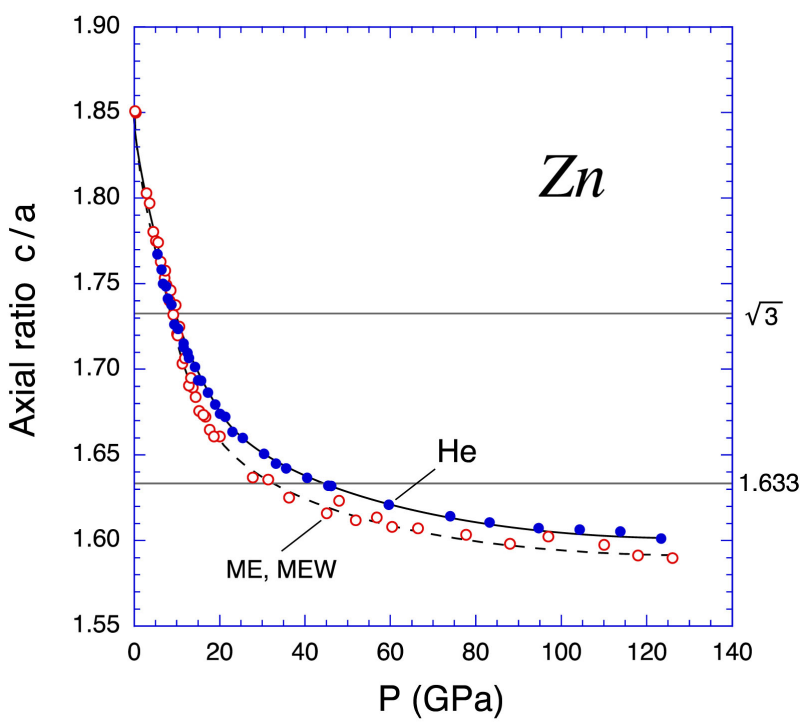

Figure 3. The change with pressure of the axial ratio $c / a$ of $\mathrm{Zn}$ at room temperature. Blue closed circles show the data taken with a He-PTM, while red open circles are taken with a ME- or MEW-PTM. The curves are drawn to guide the eyes.

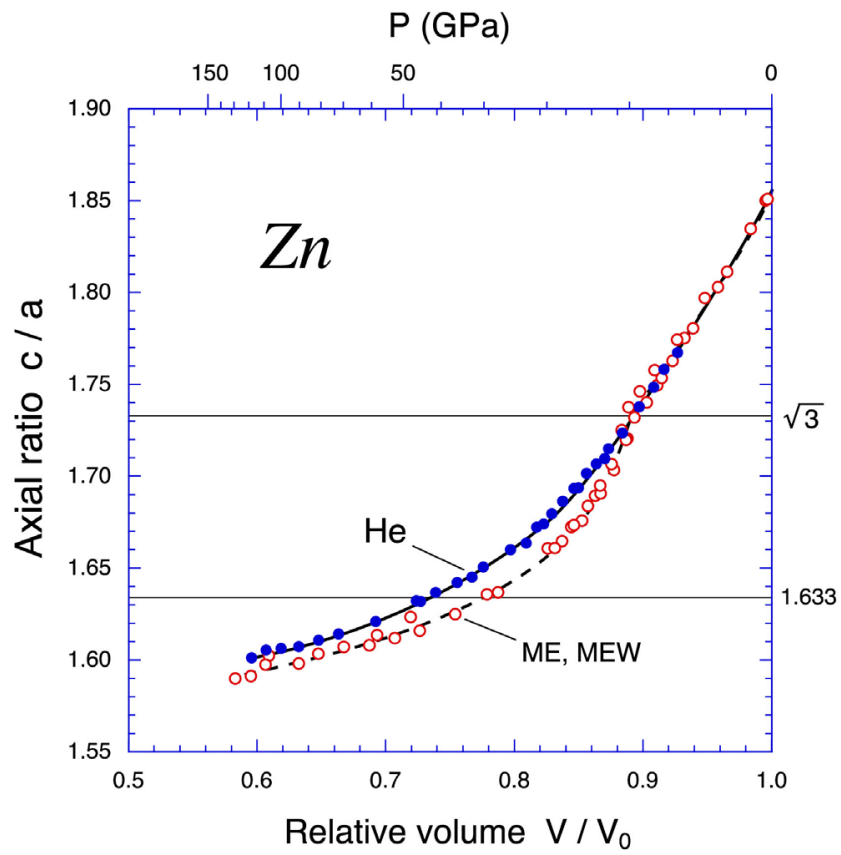

Figure 4. The change of the axial ratio $c / a$ of $\mathrm{Zn}$ as a function of relative volume $V / V_{0}$ at room temperature. Corresponding pressures are shown on the upper horizontal axis. Blue closed circles show the data taken with a He-PTM, while red open circles are taken with a ME- or MEW-PTM.

data taken with He-PTM (run 7, 9, and 10, see Table A1), we judge that the data with $\mathrm{He}$ are more reliable and those with other PTM (ME and MEW) suffer from the influence of nonhydrostaticity. A part from the larger scatter of the data, one notices a change in the curvature for the data with ME- and MEW-PTM at around $V / V_{0}=0.9$ or $c / a=1.73(\sim \sqrt{3})$. This is the so-called " $c / a$ anomaly", 
which was once interpreted as a signature of the ETT or a singularity in the hcp structure [20]. However, as already mentioned in the preceding section, this anomaly was most probably induced by the solidification of the PTM. See [23] for further details of the possible mechanism of the emergence of anomaly. Finally, we see that the $c / a$ axial ratio passes through 1.633 , the value for ideal hexagonal close-packing, without any anomaly. This occurs at around $V / V_{0}=$ 0.73 and $P=45 \mathrm{GPa}$ for the case of He-PTM.

Figure 5 shows the comparison of the pressure dependence of the lattice parameters at room and low temperatures. Although the low-temperature data were taken at different temperatures in the range $27-58 \mathrm{~K}$ (see Table A2 in Appendix), it causes negligible effect on the lattice parameters, since the thermal expansion of $\mathrm{Zn}$ is small enough below $100 \mathrm{~K}$ [6]. He-PTM was used for all the data. In the low-temperature experiments, care was taken to change pressure at elevated temperature and then cool down the cell to the target temperature every time [25]. It is well known that if one changes pressure at low temperature with a He-PTM, large nonhydrostatic stress appears [43]. In Figure 5 we clearly see that the difference of the lattice parameters at room and low temperatures gradually decreases at high pressures: the thermal expansion of $\mathrm{Zn}$ decreases with pressure at least in the low-temperature region. The difference of the lattice parameters at room and low temperatures becomes zero at about $15 \mathrm{GPa}$. At higher pressures, the thermal expansion of the a-axis may become negative, but we need further careful experiments to prove it.

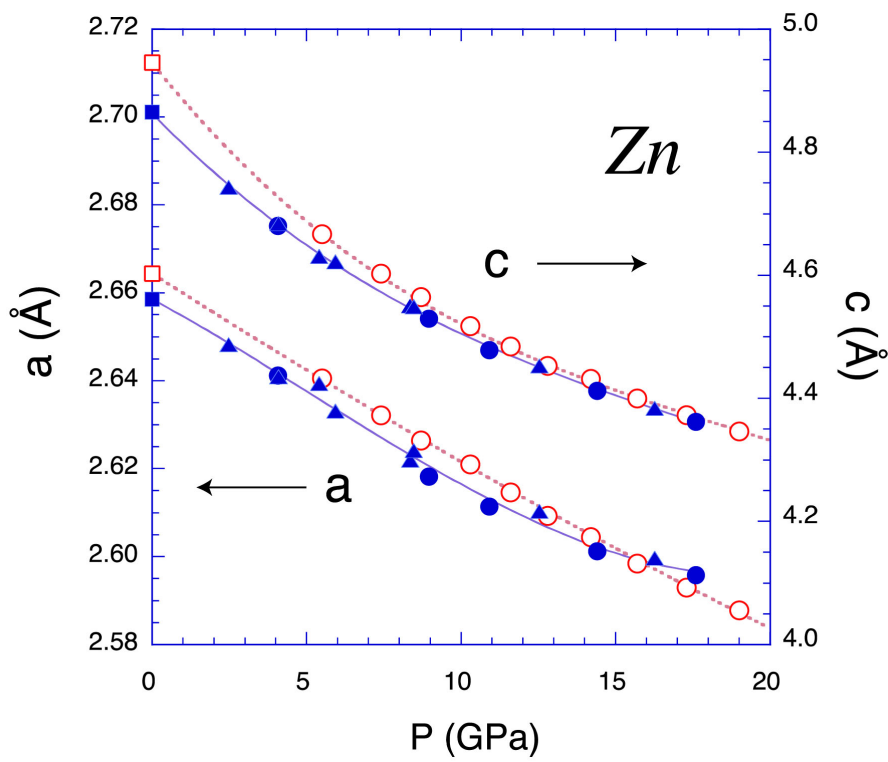

Figure 5. Lattice parameters of $\mathrm{Zn}$ at high pressures at $297 \mathrm{~K}$ (red open symbols) and at $40 \mathrm{~K}$ (blue closed symbols). Circles and triangles show the data in different experimental runs at low temperature. All the data were taken with a He-PTM. The data at atmospheric pressure and at $297 \mathrm{~K}$ are taken from [2]. Those at $40 \mathrm{~K}$ are estimated by combining the thermal-expansion data near room temperature [42] with those at low temperature [6]. Note that different scales are used for the $a$ and $c$ axes. This figure was adapted from Figure 2 of [24]. 


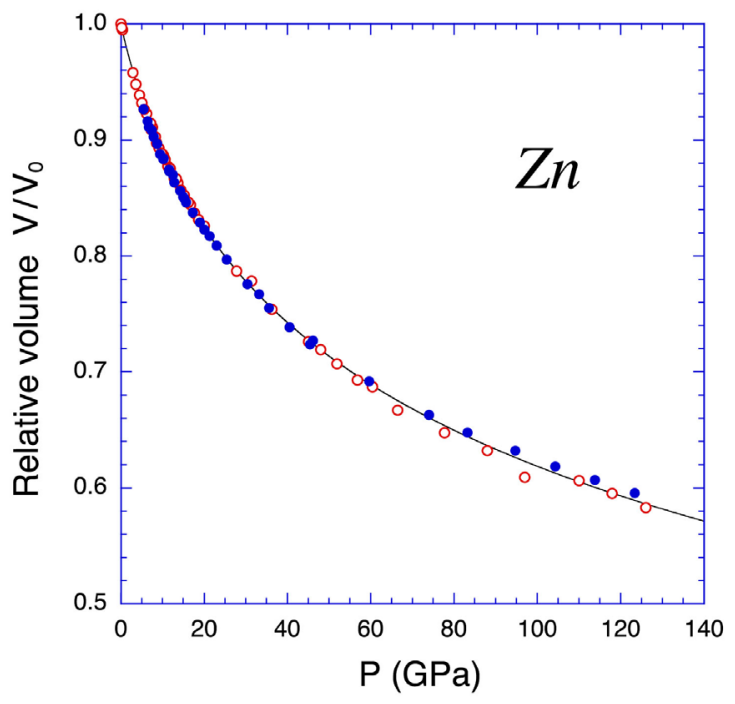

Figure 6. Relative volume of $\mathrm{Zn}$ as a function of pressure at room temperature. Blue closed circles show the data taken with a He-PTM, while red open circles are taken with a ME- or a MEW-PTM. The solid curve is a fit to all the data with the Vinet form of the equation of state with $B_{0}=63 \mathrm{GPa}$ and $B_{0}{ }^{\prime}=5.3$.

Table 2. Bulk modulus $B_{0}$ and its pressure derivative $B_{0}$ ' of $\mathrm{Zn}$ at atmospheric pressure.

\begin{tabular}{cccc}
\hline$B_{0}(\mathrm{GPa})$ & $B_{0}{ }^{\prime}$ & $P_{\max }(\mathrm{GPa})$ & Ref. \\
\hline 59.791 & 4.880 & 4.5 & {$[8]$} \\
$57(2)$ & $7.4(7)$ & 8.8 & {$[29]$} \\
$56(1)$ & $6.6(3)$ & 15 & {$[22]$} \\
$63(2)$ & $5.6(4)$ & 16 & {$[12]$} \\
$56(2)$ & $5(1)$ & 32 & {$[19]$} \\
$63(2)$ & $5.2(7)$ & 74 & {$[21]$} \\
$65(2)$ & $4.6(5)$ & 126 & present work \\
$63(4)$ & $5.3(2)$ & 126 &
\end{tabular}

Figure 6 shows the pressure-volume relationship of $\mathrm{Zn}$. We have fitted the Vinet form of the equation of state [44] to our data and obtained the bulk modulus $B_{0}$ and its pressure derivative $B_{0}$ 'at atmospheric pressure as $B_{0}=63 \pm 4 \mathrm{GPa}$ and $B_{0}^{\prime}=5.3 \pm 2$. These values are in good agreement with previous data from the literature (Table 2).

\section{Discussion}

Debates still continue about the following three issues: 1) whether any structural anomaly exists in $\mathrm{Zn}$ under high pressure, 2) whether ETTs occur in Zn under high pressure, and 3) if so, whether the ETTs accompany any detectable anomalies in physical properties. Figure 7 compares the variation of the $c / a$ axial ratio with pressure at room temperature obtained by various $\mathrm{x}$-ray diffraction experiments. The $c / a$ axial ratio is plotted as a function of relative volume $V / V_{0}$ so that the error in pressure determination is eliminated. Except for the data by 
Lynch and Drickamer [16], other data are mostly consistent at least up to about $10 \mathrm{GPa}\left(V / V_{0} \geq 0.89\right)$. At higher pressures, however, one notices discernible differences among each data set. Early data by McWhan [18] and by Schulte, Nikolaenko and Holzapfel [19] as well as our data with ME-and MEW-PTM show smaller $c /$ a ratio, whereas the recent data by Errandonea et al. [12] show an upward shift. Our data with He-PTM smoothly change without any anomaly. The He-PTM provides the best quasi-hydrostatic conditions at least in the pressure range shown here (from the solidification pressure of $11.5 \mathrm{GPa}$ to $25 \mathrm{GPa}$ ). It is therefore reasonable to assume that the $c /$ a ratio smoothly changes with pressure under hydrostatic condition. The systematic deviation of other data would be due to the effect of nonhydrostatic stress of the solid or solidified PTM as has been demonstrated in the case of Nb compressed with a MEW-PTM [45]. Our data with a He-PTM seem to exclude any anomaly. However, this is governed by the experimental precision. The error of lattice parameters in the least-squares fitting was about $\pm 0.01 \%-0.02 \%$ for our He data at room temperature (Table A1). The scatter of the data among three experimental runs with a He-PTM was, on the other hand, much larger than this and amounts to about $\pm 0.2 \%$ or \pm 0.003 in the $c / a$ ratio as indicated by the gray curve in Figure 7.

A number of theoretical calculations have been done on the change of the electronic structure of $\mathrm{Zn}$ under high pressure [46]-[60]. Since theoretical calculations are done for the state at $0 \mathrm{~K}$, it is better to compare them with experiments conducted at low temperature. Figure 8 compares our experimental data of the variation of the $c / a$ ratio with pressure at $40 \mathrm{~K}$ with theoretical calculations. Although the errors in the least-squares fitting of the lattice parameters are

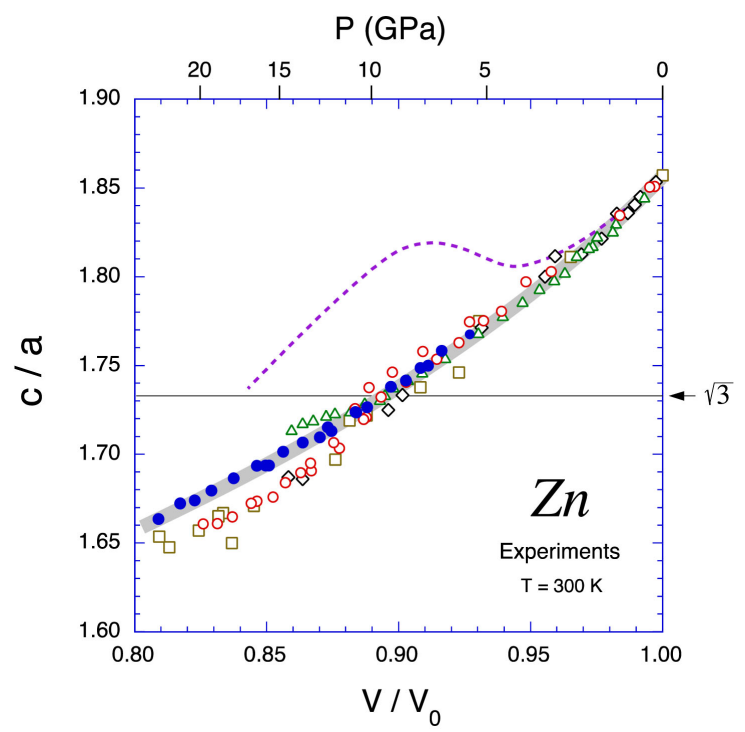

Figure 7. Variation of the $c / a$ axial ratio of $\mathrm{Zn}$ under pressure experimentally determined at room temperature. The $c / a$ axial ratio is plotted as a function of relative volume $V / V_{0}$ so that the uncertainty in pressure determination is eliminated. Circles show present data with a He-PTM (blue closed) and a ME- or a MEW-PTM (red open). Other symbols show data from the literature: triangles [12], squares [19], diamonds [18], and broken line [16]. Experimental uncertainty in $c / a$ of our He-data is indicated by a gray curve. 


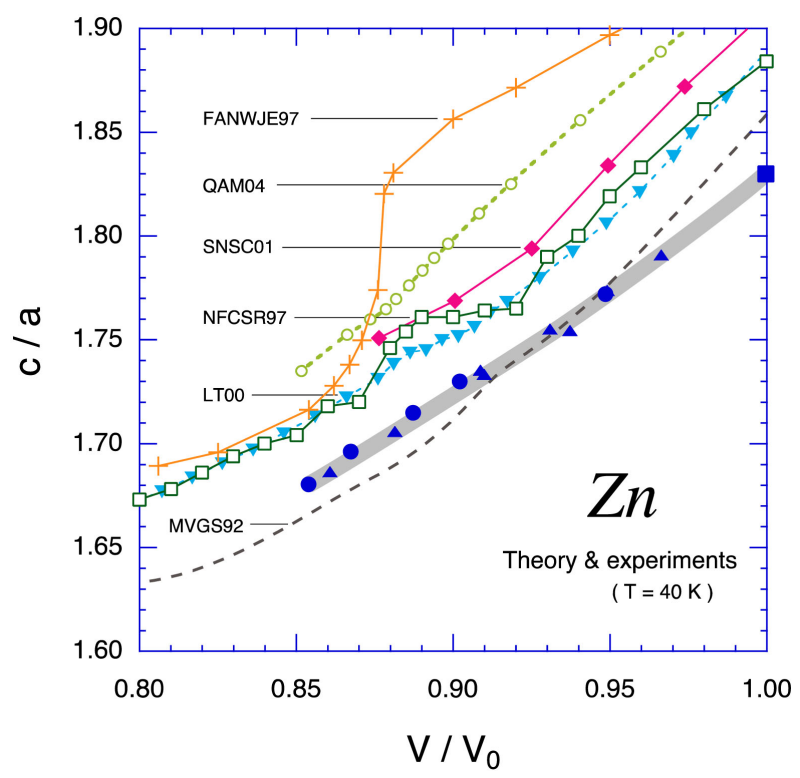

Figure 8. Variation of the $c / a$ axial ratio of $\mathrm{Zn}$ at low temperature as a function of relative volume $V / V_{0}$. Blue circles and triangles show our experimental data in two runs (run 11 and 12 given in Table A2) conducted at $40 \mathrm{~K}$ with a He-PTM. Blue closed square shows the data at atmospheric pressure from the literature [6] [42]. Other curves with labels show theoretical calculations: FANWJE97 [47], QAM04 [56], SNSC01 [51], NFCSR97 [48], LT00 [50], MVGS92 [46]. Thick gray curve indicates experimental uncertainty in $c / a$ in our experiments.

on the order of $\pm 0.01 \%$, the scatter between two experimental runs at low temperature is about $\pm 0.3 \%$ or \pm 0.005 in $c$ a as indicated by the gray curve. Compared with the present experimental results, early calculations [46] [47] [48] [50] predicted large anomalies, which should be well detected within our experimental uncertainty. Later calculations, on the other hand, showed very small [56] or even no [51] anomalies, which are comparable to our experimental uncertainties. It is clear that further experimental efforts are necessary to get structural data with much higher precision at low temperature. We emphasize that the change of the axial ratio with pressure is quite sensitive to nonhydrostatic stress. As shown in Figure 4, nonhydrostatic data largely deviate from hydrostatic (or quasi-hydrostatic) data, far exceeding the uncertainty of $\pm 0.01 \%$ in the least-squares fitting. This could also be the case for other experimental data, for which various solid PTM were used ( $\mathrm{B}+\mathrm{LiH}$ [16], $\mathrm{NaCl}$ [18], $\mathrm{Ag}_{2} \mathrm{SO}_{4}$ [27] [28], $\mathrm{N}_{2}$ or mineral oil [19] [20], $\mathrm{Pb}$ [29] [30]). The most recent experiment by Errandonea et al. shows that the $c / a$ anomaly exists at $c / a=\sqrt{3}$ both at room and high temperatures [12]. Since they used $\mathrm{NaCl}$ as a PTM, some effects of nonhydrostatic stress could be present in their experiments at room temperature.

The accuracy in theoretical calculations also needs further improvement. It has been argued that the calculated anomaly severely depends on the computational details, including the choice of the functional, the number of $k$-points sampling in the Brillouin zone, and the treatment of electron correlation with the filled $3 d$ shell [51] [56] [58] [59] [60]. For instance, Steinle-Neumann et al. 
found that if one increases the number of $k$-points sampling, the calculated anomaly disappears [51]. On the other hand, Qui et al. found no significant influence of the number of $k$-points sampling on the anomaly [56]. It is hard to prove that anomalies do not exist, because it is always limited by the precision both in experiment and theoretical calculation. We can presently say that the anomaly, if exists, does not exceed our experimental precision, $\pm 0.3 \%$ in the $c /$ a axial ratio.

Regarding to the second issue, it is quite reasonable to expect a topological change in the Fermi surface under high pressure for anisotropic substances like Zn. Early high-pressure studies focused on it by using direct probes such as magneto-resistance and de Haas-van Alphen oscillations although the pressure range was limited (Table 1). It is necessary to extend pressure to higher region and pinpoint the predicted ETTs in Zn. If the ETTs are confirmed by experiment, the next step would be to look for any anomaly in other physical properties at the pressure. The ETT under high pressure is one of the hot topics in high-pressure physics. Although a number of reports appeared to suggest the existence of ETT, for example, in Cd [61] and Os [62] [63], they are still based on indirect evidences. Direct experimental study of the band structure and the Fermi surface under pressure are highly required.

Finally, the general behavior of hcp metals under high pressure should be commented upon. The structural anisotropy of $\mathrm{Zn}$, which is evident in its large cla axial ratio at atmospheric pressure, decreases and disappears at high pressures. Similar changes were also observed in other group 12 elements, $\mathrm{Cd}$ and Hg under high pressures [4] [22]. Figure 9 shows the change of the $c / a$ axial ratio with pressure for hcp elemental metals. One can see that the axial ratios of

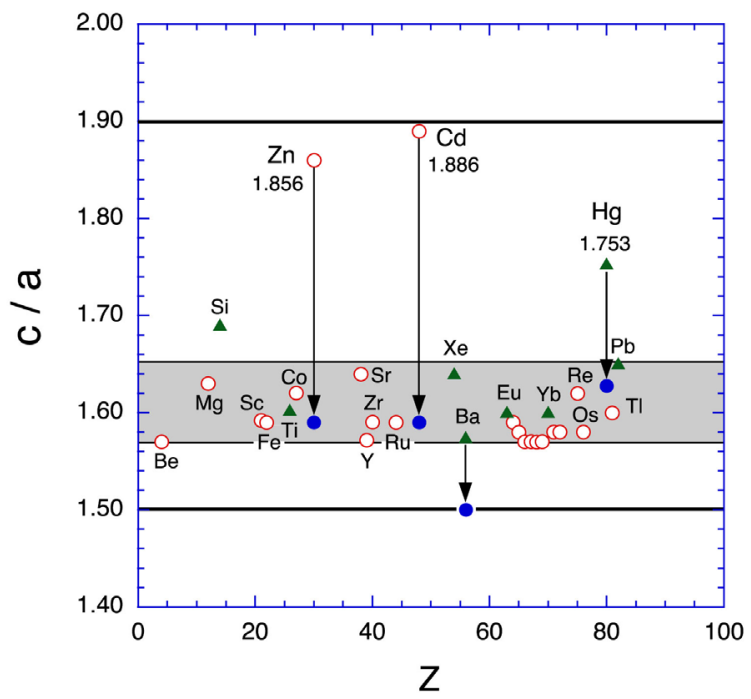

Figure 9. The $c / a$ axial ratio of hcp elemental metals. Open circles show the values at atmospheric pressure, while closed circles are the values at high pressures. Solid triangles indicate the axial ratios of the high-pressure hcp phases of some elements at the pressures where they appear. Thick solid lines show the approximate upper and lower bounds for the axial ratios of known hcp structures. The axial ratios of most hcp elemental metals fall in the range $1.57-1.65$ at high pressures as shown by the gray area. 
almost all the hcp metals fall in the range $1.57-1.65$ at high pressures. An exception is the high-pressure hcp phase II of Ba [64], in which the axial ratio continuously deviates with pressure from this range and reaches 1.50 before transforming to the next high-pressure phase. We thus infer that the possible range of the $c / a$ axial ratio for the hcp structure is approximately $1.50-1.90$. This is also in accord with the trend observed for some hcp alloys under ambient conditions [65].

\section{Conclusion}

We have extensively studied the structural change of $\mathrm{Zn}$ under high pressure up to $126 \mathrm{GPa}$ at room temperature $(297 \mathrm{~K})$ and to $18 \mathrm{GPa}$ at low temperature (40 $\mathrm{K})$ by using powder $\mathrm{x}$-ray diffraction techniques. The structural anisotropy of $\mathrm{Zn}$ rapidly decreases under high pressure as exemplified by the decrease of the $c / a$ axial ratio from 1.856 at atmospheric pressure to 1.59 at $126 \mathrm{GPa}$. Our quasi-hydrostatic data taken with a He-PTM showed no evidence of anomaly in the change of the $c / a$ axial ratio with pressure within the experimental uncertainty of $\pm 0.3 \%$ in the $c / a$ axial ratio. Further experimental and theoretical studies are necessary to identify the ETT expected for Zn under high pressure and its possible influences on the lattice properties.

\section{Acknowledgements}

The author thanks H. Fujihisa, H. Yamawaki, and T. Kikegawa for their collaboration in the high-pressure powder $\mathrm{x}$-ray diffraction experiments at the Photon Factory and thanks R. Kumai for his encouragement of writing the present paper. The diffraction experiments have been done under Proposal Nos. 93G105, 95G138, 97G269, and 99G204 of the Photon Factory, when the author worked at National Institute for Materials Science.

\section{Conflicts of Interest}

The author declares no conflicts of interest regarding the publication of this paper.

\section{References}

[1] Haynes, W.M. (2012) CRC Handbook of Chemistry and Physics. 93rd Edition, CRC Press, Boca Raton, FL.

[2] Donohue, J. (1974) The Structures of the Elements. John Wiley \& Sons Ltd., New York.

[3] Schulte, O. and Holzapfel, W.B. (1988) A New Structure of Mercury under Pressure. Physics Letters A, 131, 38-40.

[4] Takemura, K., Nakano, S., Ohishi, Y., Nakamoto, Y. and Fujihisa, H. (2015) High-Pressure Structural Study of Solid Mercury up to $200 \mathrm{GPa}$. Materials Research Express, 2, Article ID: 016502. https://doi.org/10.1088/2053-1591/2/1/016502

[5] Alers, G.A. and Neighbours, J.R. (1958) The Elastic Constants of Zinc between $4.2^{\circ}$ and $670^{\circ} \mathrm{K}$. Journal of Physics and Chemistry of Solids, 7, 58-64. 
https://doi.org/10.1016/0022-3697(58)90180-X

[6] McCammon, R.D. and White, G.K. (1965) Thermal Expansion at Low Temperatures of Hexagonal Metals: $\mathrm{Mg}, \mathrm{Zn}$ and $\mathrm{Cd}$. The Philosophical Magazine, 11, 1125-1134. https://doi.org/10.1080/14786436508224923

[7] Bridgman, P.W. (1941) Compressions and Polymorphic Transitions of Seventeen Elements to $100,000 \mathrm{~kg} / \mathrm{cm}^{2}$. Physical Review, 60, 351-354. https://doi.org/10.1103/PhysRev.60.351

[8] Vaidya, S.N. and Kennedy, G.C. (1970) Compressibility of 18 Metals to 45 kbar. Journal of Physics and Chemistry of Solids, 31, 2329-2345. https://doi.org/10.1016/0022-3697(70)90247-7

[9] McQueen, R.G. and Marsh, S.P. (1960) Equation of State for Nineteen Metallic Elements from Shock-Wave Measurements to Two Megabars. Journal of Applied Physics, 31, 1253-1269. https://doi.org/10.1063/1.1735815

[10] Al'tshuler, L.A., Bakanova, A.A. and Trunin, R.F. (1962) Shock Adiabats and Zero Isotherms of Seven Metals at High Pressure. Soviet Physics JETP, 15, 65-74.

[11] Akella, J., Ganguly, J., Grover, R. and Kennedy, G. (1973) Melting of Lead and Zinc to 60 kbar. Journal of Physics and Chemistry of Solids, 34, 631-636. https://doi.org/10.1016/S0022-3697(73)80168-4

[12] Errandonea, D., MacLeod, S.G., Ruiz-Fuertes, J., Burakovsky, L., McMahon, M.I., Wilson, C.W., Ibañez, J., Daisenberger, D. and Popescu, C. (2018) High-Pressure/High-Temperature Phase Diagram of Zinc. Journal of Physics. Condensed Matter, 30, Article ID: 295402. https://doi.org/10.1088/1361-648X/aacac0

[13] Gaidukov, Y.P. and Itskevich, E.S. (1964) Effect of Pressure on the Fermi Surfaces of Zinc and Cadmium. Soviet Physics JETP, 18, 51-58.

[14] Schirber, J.E. (1965) Effect of Pressure and Magnetic Field on the Connectivity of the Fermi Surface of Zinc. Physical Review, 140, A2065-A2075. https://doi.org/10.1103/PhysRev.140.A2065

[15] O’Sullivan, W.J. and Schirber, J.E. (1966) Pressure Dependence of the Low-Frequency de Haas-Van Alphen Oscillations in Zn. Physical Review, 151, 484-494. https://doi.org/10.1103/PhysRev.151.484

[16] Lynch, R.W. and Drickamer, H.G. (1965) The Effect of Pressure on the Resistance and Lattice Parameters of Cadmium and Zinc. Journal of Physics and Chemistry of Solids, 26, 63-68. ttps://doi.org/10.1016/0022-3697(65)90073-9

[17] Garg, A.B., Vijayakumar, V., Modak, P., Gaitonde, D.M., Rao, R.S., Godwal, B.K. and Sikka, S.K. (2002) High-Pressure Resistance and Equation-of-State Anomalies in Zn: A Possible Lifshitz Transition. Journal of Physics: Condensed Matter, 14, 8795-8802. https://doi.org/10.1088/0953-8984/14/38/304

[18] McWhan, D.B. (1965) Compressibility of Cadmium and Zinc to 100 kbar. Journal of Applied Physics, 36, 664-665. https://doi.org/10.1063/1.1714059

[19] Schulte, O., Nikolaenko, A. and Holzapfel, W.B. (1991) Pressure-Volume Relations for $\mathrm{Zn}, \mathrm{Cd}, \mathrm{Ga}$, In and $\mathrm{Tl}$ at Room Temperature to $30 \mathrm{GPa}$ and above. High Pressure Research, 6, 169-182.

[20] Takemura, K. (1995) Zn under Pressure: A Singularity in the hcp Structure at $c / a=$ $\sqrt{3}$. Physical Review Letters, 75, 1807-1810. https://doi.org/10.1103/PhysRevLett.75.1807

[21] Schulte, O. and Holzapfel, W.B. (1996) Effect of Pressure on the Atomic Volume of Zn Cd, and Hg up to $75 \mathrm{GPa}$. Physical Review B, 53, 569-580.

https://doi.org/10.1103/PhysRevB.53.569 
[22] Takemura, K. (1997) Structural Study of Zn and Cd to Ultrahigh Pressures. Physical Review B, 56, 5170-5179. https://doi.org/10.1103/PhysRevB.56.5170

[23] Takemura, K. (1999) Absence of the c/a Anomaly in Zn under High Pressure with a Helium-Pressure Medium. Physical Review B, 60, 6171-6174. https://doi.org/10.1103/PhysRevB.60.6171

[24] Takemura, K., Yamawaki, H., Fujihisa, H. and Kikegawa, T. (2002) Axial Ratio of $\mathrm{Zn}$ at High Pressure and Low Temperature. Physical Review B, 65, Article ID: 132107. https://doi.org/10.1103/PhysRevB.65.132107

[25] Takemura, K., Yamawaki, H., Fujihisa, H. and Kikegawa, T. (2002) High-Pressure Powder X-ray Diffraction Experiments on Zn at Low Temperature. Journal of Physics: Condensed Matter, 14, 10563-10568. https://doi.org/10.1088/0953-8984/14/44/333

[26] Takemura, K., Yamawaki, H., Fujihisa, H. and Kikegawa, T. (2002) High-Pressure $\mathrm{X}$-ray Studies of $\mathrm{Zn}$ at Room and Low Temperatures with a He-Pressure Medium. High Pressure Research, 22, 337-341. https://doi.org/10.1080/08957950212816

[27] Potzel, W., Steiner, M., Karzel, H., Schiessl, W., Köfferlein, M., Kalvius, G.M. and Blaha, P. (1995) Electronically Driven Soft Modes in Zinc Metal. Physical Review Letters, 74, 1139-1142. https://doi.org/10.1103/PhysRevLett.74.1139

[28] Steiner, M., Potzel, W., Karzel, H., Schiessl, W., Köfferlein, M., Kalvius, G.M. and Blaha, P. (1996) Electronic Topological Transition in Zinc Metal at High External Pressure. Journal of Physics: Condensed Matter, 8, 3581-3599. https://doi.org/10.1088/0953-8984/8/20/006

[29] Morgan, J.G., Von Dreele, R.B., Wochner, P. and Shapiro, S.M. (1996) Inelastic Neutron Scattering from Single Crystal Zn under High Pressure. Physical Review B, 54, 812-818. https://doi.org/10.1103/PhysRevB.54.812

[30] Klotz, S., Braden, M. and Besson, J.M. (1998) Is There an Electronic Topological Transition in Zinc under High Pressure? Physical Review Letters, 81, 1239-1242. https://doi.org/10.1103/PhysRevLett.81.1239

[31] Olijnyk, H. (1992) Raman Scattering in Metals up to 50 GPa. High Pressure Research, 10, 461-464. https://doi.org/10.1080/08957959208201457

[32] Olijnyk, H., Jephcoat, A.P., Novikov, D.L. and Christensen, N.E. (2000) Pressure Shift of the Zone-Center TO Mode of Zn. Physical Review B, 62, 5508-5512. https://doi.org/10.1103/PhysRevB.62.5508

[33] Aquilanti, G., Trapananti, A., Minicucci, M., Liscio, F., Twaróg, A., Principi, E. and Pascarelli, S. (2007) Electronic Topological Transition in Zinc under Pressure: An X-ray Absorption Spectroscopy Study. Physical Review B, 76, Article ID: 144102. https://doi.org/10.1103/PhysRevB.76.144102

[34] Goodenough, J.B. (1953) A Theory of the Deviation from Close Packing in Hexagonal Metal Crystals. Physical Review, 89, 282-294. https://doi.org/10.1103/PhysRev.89.282

[35] Lifshitz, I.M. (1960) Anomalies of Electron Characteristics of a Metal in the High Pressure Region. Soviet Physics JETP, 11, 1130-1135.

[36] Takemura, K. (2001) Evaluation of the Hydrostaticity of a Helium-Pressure Medium with Powder X-Ray Diffraction Techniques. Journal of Applied Physics, 89, 662-668. https://doi.org/10.1063/1.1328410

[37] Piermarini, G.J., Block, S. and Barnett, J.D. (1973) Hydrostatic Limits in Liquids and Solids to $100 \mathrm{kbar}$. Journal of Applied Physics, 44, 5377-5382.

https://doi.org/10.1063/1.1662159 
[38] Nakamura, Y., Fujishiro, I. and Taniguchi, K. (1991) Hysteresis of Ruby Fluorescent Line by Pressure and Annealing Effect. High Pressure Research, 6, 301-307. https://doi.org/10.1080/08957959108203215

[39] Bell P.M. and Mao, H.K. (1981) Degrees of Hydrostaticity in He, Ne, and Ar Pressure-Transmitting Media. Carnegie Institution of Washington Yearbook, 80, 404-406.

[40] Mao, H.K., Bell, P.M., Shaner, J.W. and Steinberg, D.J. (1978) Specific Volume Measurements of $\mathrm{Cu}, \mathrm{Pd}$, and $\mathrm{Ag}$ and Calibration of the Ruby $R_{1}$ Fluorescence Pressure Gauge from 0.06 to 1 Mbar. Journal of Applied Physics, 49, 3276-3283. https://doi.org/10.1063/1.325277

[41] Zha, C.-S., Mao, H.-K. and Hemley, R.J. (2000) Elasticity of MgO and a Primary Pressure Scale to $55 \mathrm{GPa}$. Proceedings of the National Academy of Sciences of the United States of America, 97, 13494-13499. https://doi.org/10.1073/pnas.240466697

[42] Owen, E.A. and Yates, E.L. (1934) The Thermal Expansion of the Crystal Lattices of Silver, Platinum, and Zinc. Philosophical Magazine, 17, 113-131. https://doi.org/10.1080/14786443409462374

[43] Reimann, K. (1996) Two- and Three-Photon Spectroscopy of Solids under High Pressure. High Pressure Research, 15, 73-93. https://doi.org/10.1080/08957959608240462

[44] Vinet, P., Ferrante, J., Rose, J.H. and Smith, J.R. (1987) Compressibility of Solids. Journal of Geophysical Research, 92, 9319-9325. https://doi.org/10.1029/JB092iB09p09319

[45] Takemura, K. and Singh, A.K. (2006) High-Pressure Equation of State for Nb with a Helium-Pressure Medium: Powder X-Ray Diffraction Experiments. Physical Review $B$, 73, Article ID: 224119. https://doi.org/10.1103/PhysRevB.73.224119

[46] Meenakshi, S., Vijayakumar, V., Godwal, B.K. and Sikka, S.K. (1992) Distorted HCP Structure of Zinc under Pressure. Physical Review B, 46, 14359-14361. https://doi.org/10.1103/PhysRevB.46.14359

[47] Fast, L., Ahuja, R., Nordström, L., Wills, J.M., Johansson, B. and Eriksson, O. (1997) Anomaly in cla Ratio of Zn under Pressure. Physical Review Letters, 79, 2301-2303. https://doi.org/10.1103/PhysRevLett.79.2301

[48] Novikov, D.L., Freeman, A.J., Christensen, N.E., Svane, A. and Rodriguez, C.O. (1997) LDA Simulations of Pressure-Induced Anomalies in $c / a$ and Electric-Field Gradients for Zn and Cd. Physical Review B, 56, 7206-7214. https://doi.org/10.1103/PhysRevB.56.7206

[49] Novikov, D.L., Katsnelson, M.I., Trefilov, A.V., Freeman, A.J., Christensen, N.E., Svane, A. and Rodriguez, C.O. (1999) Anisotropy of Thermal Expansions and Electronic Topological Transitions in Zn and Cd under Pressure. Physical Review B, 59, 4557-4560. https://doi.org/10.1103/PhysRevB.59.4557

[50] Li, Z. and Tse, J.S. (2000) Phonon Anomaly in High-Pressure Zn. Physical Review Letters, 85, 5130-5133. https://doi.org/10.1103/PhysRevLett.85.5130

[51] Steinle-Neumann, G., Stixrude, L. and Cohen, R.E. (2001) Absence of Lattice Strain Anomalies at the Electronic Topological Transition in Zinc at High Pressure. Physical Review B, 63, Article ID: 054103. https://doi.org/10.1103/PhysRevB.63.054103

[52] Kechin, V.V. (2001) Electronic Topological Transitions in Zn under Compression. Physical Review B, 63, Article ID: 045119. https://doi.org/10.1103/PhysRevB.63.045119

[53] Rao, R.S., Modak, P. and Godwal, B.K. (2001) Comment on "Phonon Anomaly in High-Pressure Zn”. Physical Review Letters, 87, Article ID: 259601. 
https://doi.org/10.1103/PhysRevLett.87.259601

[54] Li, Z. and Tse, J.S. (2001) Li and Tse Reply. Physical Review Letters, 87, Article ID: 2596021. https://doi.org/10.1103/PhysRevLett.87.259602

[55] Qiu, S.L. and Marcus, P.M. (2003) First-Principles Derivation of Structural Anomalies in hcp $\mathrm{Zn}$ and hcp Fe under Pressure. Journal of Physics. Condensed Matter, 15, L755-L761. https://doi.org/10.1088/0953-8984/15/50/L02

[56] Qiu, S.L., Apostol, F. and Marcus, P.M. (2004) Structural Anomalies in HCP Metals under Pressure: Zn and Cd. Journal of Physics: Condensed Matter, 16, 6405-6414. https://doi.org/10.1088/0953-8984/16/36/007.

[57] Qiu, S.L., Apostol, F. and Marcus, P.M. (2005) Pressure Dependence of the TO Phonon Frequency in HCP Zn. Journal of Physics. Condensed Matter, 17, 2121-2128. https://doi.org/10.1088/0953-8984/17/13/010

[58] Wedig, U., Jansen, M., Paulus, B., Rosciszewski, K. and Sony, P. (2007) Structural and Electronic Properties of $\mathrm{Mg}, \mathrm{Zn}$, and Cd from Hartree-Fock and Density Functional Calculations Including Hybrid Functionals. Physical Review B, 75, Article ID: 205123. https://doi.org/10.1103/PhysRevB.75.205123

[59] Gaston, N., Paulus, B., Wedig, U. and Jansen, M. (2008) Multiple Minima on the Energy Landscape of Elemental Zinc: A Wave Function Based Ab Initio Study. Physical Review Letters, 100, Article ID: 226404. https://doi.org/10.1103/PhysRevLett.100.226404

[60] Gaston, N., Andrae, D., Paulus, B., Wedig, U. and Jansen, M. (2010) Understanding the hcp Anisotropy in Cd and $\mathrm{Zn}$ : The Role of Electron Correlation in Determining the Potential Energy Surface. Physical Chemistry Chemical Physics, 12, 681-687. https://doi.org/10.1039/B915967C

[61] Pratesi, G., Di Cicco, A., Minicucci, M. and Itiè, J.-P. (2005) Anomalies in the Structure of Solid Cd under Pressure: An X-ray Diffraction Study. Journal of Physics. Condensed Matter, 17, 2625-2632. https://doi.org/10.1088/0953-8984/17/17/010

[62] Occelli, F., et al. (2004) Experimental Evidence for a High-Pressure Isostructural Phase Transition in Osmium. Physical Review Letters, 93, Article ID: 095502. https://doi.org/10.1103/PhysRevLett.93.095502

[63] Dubrovinsky, L., et al. (2015) The Most Incompressible Metal Osmium at Static Pressures above 750 Gigapascals. Nature, 525, 226-229. https://doi.org/10.1038/nature14681

[64] Takemura, K. (1994) High-Pressure Structural Study of Barium to 90 GPa. Physical Review B, 50, 16238-16246. https://doi.org/10.1103/PhysRevB.50.16238

[65] Massalski, T.B. (1962) Lattice Spacing Trends in Close-Packed Hexagonal Phases Based on the Noble Metals. Le Journal de Physique et le Radium, 23, 647-654. 


\section{Appendix}

Table A1 and Table A2 summarize the structural data of $\mathrm{Zn}$ under high pressure at room and low temperatures, respectively.

Table A1. Lattice parameters, axial ratio, and relative volume of $\mathrm{Zn}$ under high pressure at room temperature $(297 \mathrm{~K})$. The errors in $a, c, c / a$, and $V / V_{0}$ given in pharenseses are from least-squares fitting. Relative volume was calculated against the volume at atmospheric pressure by using the lattice parameters $a_{0}=2.6644$ (3) $\AA$ and $c_{0}=4.9454$ (3) $\AA$ from the literature [2]. Different pressure-transmitting media (PTM) were used in each experimental run as indicated on the second column: methanol-ethanol mixture (ME), methanol-ethanol-water mixture (MEW), and helium. Data taken on decreasing pressure are shown in square brackets.

\begin{tabular}{|c|c|c|c|c|c|c|}
\hline Run & PTM & $P(\mathrm{GPa})$ & $a(\AA)$ & $c(\AA)$ & $c l a$ & $V / V_{0}$ \\
\hline \multirow[t]{9}{*}{1} & $\mathrm{ME}$ & $0.4(1)$ & $2.6631(3)$ & $4.9269(7)$ & $1.8501(3)$ & $0.9953(3)$ \\
\hline & & $7.6(1)$ & $2.6343(5)$ & $4.6087(14)$ & $1.7495(6)$ & $0.9110(4)$ \\
\hline & & $13.7(1)$ & $2.6173(7)$ & $4.4219(17)$ & $1.6895(8)$ & $0.8629(6)$ \\
\hline & & $20.0(2)$ & $2.5942(2)$ & $4.3084(5)$ & $1.6608(2)$ & $0.8259(2)$ \\
\hline & & $27.8(2)$ & $2.5653(1)$ & $4.1992(7)$ & $1.6369(3)$ & $0.7871(2)$ \\
\hline & & $36.3(2)$ & $2.5348(3)$ & $4.1193(12)$ & $1.6251(5)$ & 0.7539 \\
\hline & & $45.1(1)$ & $2.5082(3)$ & $4.0533(14)$ & $1.6160(6)$ & $0.7263(3)$ \\
\hline & & $51.9(1)$ & $2.4878(4)$ & $4.0102(18)$ & $1.6119(8)$ & $0.7070(4)$ \\
\hline & & $60.4(3)$ & $2.4665(4)$ & $3.9662(20)$ & $1.6080(9)$ & $0.6873(4)$ \\
\hline \multirow[t]{4}{*}{2} & $\mathrm{ME}$ & $4.5(1)$ & $2.6453(4)$ & $4.7100(11)$ & $1.7805(5)$ & $0.9388(4)$ \\
\hline & & $10.0(1)$ & $2.6262(4)$ & $4.5189(21)$ & $1.7207(8)$ & $0.8877(5)$ \\
\hline & & $16.7(2)$ & $2.6072(6)$ & $4.3602(34)$ & $1.6724(14)$ & $0.8442(8)$ \\
\hline & & $31.4(3)$ & $2.5565(1)$ & $4.1819(1)$ & $1.6358(1)$ & $0.7785(1)$ \\
\hline \multirow[t]{9}{*}{3} & $\mathrm{ME}$ & $48.0(1)$ & $2.4963(18)$ & $4.0524(43)$ & $1.6234(21)$ & $0.7193(13)$ \\
\hline & & $56.8(1)$ & $2.4708(17)$ & $3.9866(39)$ & $1.6135(19)$ & $0.6932(12)$ \\
\hline & & $66.5(1)$ & $2.4426(16)$ & $3.9256(38)$ & $1.6071(19)$ & $0.6671(11)$ \\
\hline & & $77.7(3)$ & $2.4202(19)$ & $3.8809(44)$ & $1.6035(22)$ & $0.6475(12)$ \\
\hline & & $88(1)$ & 2.4037 (19) & $3.8414(43)$ & $1.5981(22)$ & $0.6322(12)$ \\
\hline & & $97(1)$ & $2.3720(9)$ & $3.8011(20)$ & $1.6025(10)$ & $0.6092(6)$ \\
\hline & & $110(2)$ & $2.3707(11)$ & $3.7873(26)$ & $1.5975(13)$ & $0.6063(7)$ \\
\hline & & $118(3)$ & $2.3591(21)$ & $3.7541(47)$ & $1.5913(24)$ & $0.5951(13)$ \\
\hline & & $126(3)$ & $2.3436(15)$ & $3.7261(33)$ & $1.5899(17)$ & 0.5829 (9) \\
\hline \multirow[t]{6}{*}{4} & MEW & $2.9(1)$ & $2.6522(1)$ & $4.7820(3)$ & $1.8030(1)$ & $0.9581(1)$ \\
\hline & & $5.1(1)$ & $2.6415(1)$ & $4.6893(3)$ & $1.7752(1)$ & $0.9320(1)$ \\
\hline & & $6.2(1)$ & $2.6390(2)$ & $4.6522(5)$ & $1.7629(2)$ & 0.9229 (1) \\
\hline & & $7.1(1)$ & $2.6357(2)$ & $4.6216(5)$ & $1.7535(2)$ & 0.9145 (1) \\
\hline & & $8.3(1)$ & $2.6311(3)$ & $4.5787(11)$ & $1.7402(5)$ & 0.9029 (3) \\
\hline & & $9.2(1)$ & $2.6259(2)$ & $4.5481(4)$ & $1.7320(2)$ & $0.8933(1)$ \\
\hline
\end{tabular}




\section{Continued}

\begin{tabular}{|c|c|c|c|c|c|c|}
\hline & & $10.2(1)$ & $2.6259(4)$ & $4.5159(22)$ & $1.7198(9)$ & $0.8869(5)$ \\
\hline & & $11.3(1)$ & $2.6248(1)$ & $4.4712(7)$ & $1.7034(3)$ & $0.8774(2)$ \\
\hline & & $12.8(1)$ & 2.6209 & $4.4308(11)$ & $1.6906(4)$ & $0.8669(2)$ \\
\hline & & $15.2(1)$ & 2.6138 & $4.3803(15)$ & $1.6758(6)$ & $0.8524(3)$ \\
\hline & & {$[1.1(1)]$} & {$[2.6601(1)]$} & {$[4.8806(6)]$} & {$[1.8347(2)]$} & {$[0.9869(1)]$} \\
\hline \multirow[t]{13}{*}{5} & MEW & $0.2(1)$ & $2.6641(1)$ & $4.9310(3)$ & $1.8509(1)$ & 0.9969 (1) \\
\hline & & $3.6(1)$ & $2.6458(2)$ & $4.7548(5)$ & $1.7971(2)$ & $0.9481(2)$ \\
\hline & & $5.6(1)$ & $2.6368(2)$ & $4.6786(6)$ & $1.7743(3)$ & $0.9266(2)$ \\
\hline & & $7.3(1)$ & $2.6282(2)$ & $4.6199(4)$ & $1.7578(2)$ & $0.9090(2)$ \\
\hline & & $8.6(1)$ & $2.6230(1)$ & $4.5801(1)$ & $1.7462(1)$ & $0.8976(1)$ \\
\hline & & $9.7(1)$ & $2.6186(1)$ & $4.5501(3)$ & $1.7376(1)$ & $0.8887(1)$ \\
\hline & & $10.6(1)$ & $2.6196(3)$ & $4.5189(7)$ & $1.7250(3)$ & $0.8833(2)$ \\
\hline & & $11.9(1)$ & $2.6212(8)$ & $4.4733(20)$ & $1.7066(9)$ & $0.8754(1)$ \\
\hline & & $13.3(1)$ & $2.6184(8)$ & $4.4381(18)$ & $1.6950(8)$ & $0.8667(6)$ \\
\hline & & $14.4(1)$ & $2.6144(4)$ & $4.4025(10)$ & $1.6839(5)$ & $0.8571(4)$ \\
\hline & & $16.2(1)$ & $2.6088(3)$ & $4.3658(6)$ & $1.6735(3)$ & $0.8463(2)$ \\
\hline & & $17.7(2)$ & $2.6037(4)$ & $4.3346(10)$ & $1.6648(5)$ & $0.8370(3)$ \\
\hline & & $18.7(2)$ & $2.5997(5)$ & $4.3179(12)$ & $1.6609(6)$ & $0.8313(4)$ \\
\hline \multirow[t]{16}{*}{7} & $\mathrm{He}$ & $5.5(1)$ & $2.6405(2)$ & $4.6669(4)$ & $1.7674(2)$ & $0.9269(2)$ \\
\hline & & $7.4(1)$ & $2.6322(1)$ & $4.6026(4)$ & $1.7486(2)$ & $0.9083(2)$ \\
\hline & & $8.7(1)$ & $2.6265(2)$ & $4.5645(5)$ & $1.7379(2)$ & $0.8969(2)$ \\
\hline & & $10.3(1)$ & $2.6210(2)$ & $4.5175(7)$ & $1.7236(3)$ & $0.8840(3)$ \\
\hline & & $11.6(1)$ & $2.6146(2)$ & $4.4844(4)$ & $1.7151(2)$ & $0.8732(2)$ \\
\hline & & $12.8(1)$ & $2.6093(3)$ & $4.4533(8)$ & $1.7067(4)$ & $0.8636(4)$ \\
\hline & & $14.2(1)$ & $2.6044(3)$ & $4.4315(7)$ & $1.7015(3)$ & $0.8562(4)$ \\
\hline & & $15.7(1)$ & 2.5985 (3) & $4.4004(13)$ & $1.6935(5)$ & $0.8463(5)$ \\
\hline & & $17.3(2)$ & $2.5930(2)$ & $4.3730(10)$ & $1.6865(4)$ & $0.8375(4)$ \\
\hline & & $19.0(1)$ & $2.5878(2)$ & $4.3465(10)$ & $1.6796(4)$ & $0.8291(4)$ \\
\hline & & $21.3(1)$ & $2.5791(2)$ & $4.3131(7)$ & $1.6723(3)$ & $0.8172(3)$ \\
\hline & & {$[15.0(3)]$} & {$[2.6030(1)]$} & {$[4.4088(5)]$} & {$[1.6937(2)]$} & {$[0.8509(2)]$} \\
\hline & & {$[11.6(3)]$} & {$[2.6169(3)]$} & {$[4.4832(7)]$} & {$[1.7131(3)]$} & {$[0.8745(4)]$} \\
\hline & & [9.4 (1)] & {$[2.6236(2)]$} & [4.5294 (8)] & {$[1.7264(3)]$} & {$[0.8881(4)]$} \\
\hline & & {$[7.9(1)]$} & {$[2.6305(1)]$} & {$[4.5804(3)]$} & {$[1.7413(1)]$} & {$[0.9028(1)]$} \\
\hline & & {$[6.8(1)]$} & {$[2.6343(2)]$} & {$[4.6100(3)]$} & {$[1.7500(2)]$} & {$[0.9112(2)]$} \\
\hline \multirow[t]{4}{*}{9} & $\mathrm{He}$ & $6.4(1)$ & $2.6349(4)$ & $4.6329(9)$ & $1.7583(4)$ & $0.9162(5)$ \\
\hline & & $10.2(1)$ & $2.6203(2)$ & $4.5175(4)$ & $1.7240(2)$ & $0.8835(2)$ \\
\hline & & $15.2(1)$ & $2.6018(3)$ & $4.4066(7)$ & $1.6937(3)$ & $0.8497(4)$ \\
\hline & & $20.1(1)$ & $2.5839(1)$ & $4.3258(2)$ & $1.6741(1)$ & $0.8227(1)$ \\
\hline
\end{tabular}




\section{Continued}

\begin{tabular}{llllll}
\hline $25.4(1)$ & $2.5640(2)$ & $4.2563(4)$ & $1.6600(2)$ & $0.7970(2)$ \\
$30.4(1)$ & $2.5458(2)$ & $4.2022(4)$ & $1.6506(2)$ & $0.7758(2)$ \\
& $35.6(2)$ & $2.5276(2)$ & $4.1509(4)$ & $1.6422(2)$ & $0.7554(2)$ \\
& $40.5(3)$ & $2.5117(2)$ & $4.1110(5)$ & $1.6367(2)$ & $0.7387(2)$ \\
& $45.4(2)$ & $2.4971(5)$ & $4.0755(10)$ & $1.6321(5)$ & $0.7239(5)$ \\
& $12.5(1)$ & $2.6143(2)$ & $4.4693(4)$ & $1.7096(2)$ & $0.8701(2)$ \\
& $23.0(2)$ & $2.5750(4)$ & $4.2837(9)$ & $1.6636(4)$ & $0.8090(5)$ \\
& $33.2(1)$ & $2.5391(2)$ & $4.1770(5)$ & $1.6451(2)$ & $0.7671(2)$ \\
& $46.1(3)$ & $2.5009(9)$ & $4.0811(19)$ & $1.6319(10)$ & $0.7271(10)$ \\
& $59.6(4)$ & $2.4657(3)$ & $3.9968(6)$ & $1.6210(3)$ & $0.6921(3)$ \\
& $74.0(6)$ & $2.4341(9)$ & $3.9292(19)$ & $1.6142(10)$ & $0.6631(9)$ \\
& $83(1)$ & $2.4169(4)$ & $3.8930(8)$ & $1.6107(4)$ & $0.6477(4)$ \\
& $95(2)$ & $2.3991(14)$ & $3.8560(27)$ & $1.6073(15)$ & $0.6322(14)$ \\
& $104(2)$ & $2.3821(16)$ & $3.8265(32)$ & $1.6064(17)$ & $0.6185(16)$ \\
& $114(3)$ & $2.3676(21)$ & $3.8009(42)$ & $1.6054(23)$ & $0.6069(20)$ \\
& $123(4)$ & $2.3549(17)$ & $3.7707(34)$ & $1.6012(18)$ & $0.5956(16)$ \\
\hline
\end{tabular}

Table A2. Lattice parameters, axial ratio, and relative volume of $\mathrm{Zn}$ under high pressure at low temperature. The errors in $a, c, c / a$, and $V / V_{0}$ given in parentheses are from least-squares fitting. The PTM was helim for all experimental runs. Relative volume was calculated against the volume at atmospheric presssure and at $40 \mathrm{~K}$. This was obtained by using the lattice parameters $a_{0}=2.6586 \AA$ and $c_{0}=4.8651 \AA$ estimated from the thermal expansion data [6] [42].

\begin{tabular}{ccccccc}
\hline Run & $P(\mathrm{GPa})$ & $T(\mathrm{~K})$ & $a(\AA)$ & $c(\AA)$ & $c / a$ & $V / V_{0}$ \\
\hline 11 & $4.4(1)$ & 298 & $2.6423(2)$ & $4.6888(6)$ & $1.7745(3)$ & $0.9325(3)$ \\
& $4.1(1)$ & 35 & $2.6412(2)$ & $4.6805(6)$ & $1.7721(3)$ & $0.9300(3)$ \\
& $8.9(1)$ & 33 & $2.6182(3)$ & $4.5297(11)$ & $1.7301(5)$ & $0.8845(5)$ \\
& $10.9(1)$ & 33 & $2.6114(4)$ & $4.4784(15)$ & $1.7149(6)$ & $0.8699(6)$ \\
& $14.4(1)$ & 35 & $2.6012(3)$ & $4.4124(8)$ & $1.6963(4)$ & $0.8504(4)$ \\
12 & $17.6(1)$ & 33 & $2.5958(3)$ & $4.3620(11)$ & $1.6804(5)$ & $0.8372(5)$ \\
& $2.2(1)$ & 300 & $2.6555(3)$ & $4.8177(8)$ & $1.8142(4)$ & $0.9677(4)$ \\
& $2.5(1)$ & 36 & $2.6482(2)$ & $4.7427(7)$ & $1.7909(3)$ & $0.9474(3)$ \\
& $4.1(1)$ & 28 & $2.6409(3)$ & $4.6839(13)$ & $1.7736(5)$ & $0.9305(5)$ \\
& $5.9(1)$ & 28 & $2.6331(4)$ & $4.6216(14)$ & $1.7552(6)$ & $0.9127(6)$ \\
& $8.4(1)$ & 27 & $2.6219(3)$ & $4.5500(10)$ & $1.7354(4)$ & $0.8909(5)$ \\
& $7.2(1)$ & 300 & $2.6371(4)$ & $4.6240(13)$ & $1.7534(6)$ & $0.9159(6)$ \\
& $5.4(1)$ & 41 & $2.6394(3)$ & $4.6306(11)$ & $1.7544(5)$ & $0.9189(5)$ \\
& $8.5(1)$ & 58 & $2.6241(3)$ & $4.5485(11)$ & $1.7334(5)$ & $0.8921(5)$ \\
$12.5(1)$ & 46 & $2.6103(3)$ & $4.4528(6)$ & $1.7059(3)$ & $0.8642(4)$ \\
$16.3(1)$ & 44 & $2.5996(1)$ & $4.3840(6)$ & $1.6864(2)$ & $0.8439(2)$ \\
\hline
\end{tabular}

